\title{
LHC RadMon SRAM Detectors Used at Different Voltages to Determine the Thermal Neutron to High Energy Hadron Fluence Ratio
}

\author{
D. Kramer, M. Brugger, V. Klupak, C. Pignard, K. Roeed, G. Spiezia, L. Viererbl, and T. Wijnands
}

\begin{abstract}
The thermal neutron SEU cross-section of the Toshiba SRAM memory used in the LHC RadMon system was measured at different voltages. A method using the difference in its response compared to mixed particle energy field is proposed to be used as a discriminator between thermal neutron and high-energy hadron fluences. For test purposes, the proposed method was used at the CNGS and CERF facilities to estimate the field composition by counting SEUs at two different voltages and the results were compared to simulations.
\end{abstract}

Index Terms-Large hadron collider (LHC), radiation, SEU, SRAM, thermal neutrons.

\section{INTRODUCTION}

$\mathbf{T}$ HE main electronic systems of the large hadron collider (LHC) [1] accelerator are located in the underground areas, where partly elevated radiation levels are expected. The RadMon radiation detection network serves as an early warning system to protect the concerned electronics, to help scheduling its maintenance and to plan possible mitigation options.

\section{A. RadMon System in the LHC}

The installation consists of about 330 RadMon [2] detectors, which are providing TID measurements using RadFets of different sensitivities, $1 \mathrm{MeV}$ neutron equivalent fluence through PIN diodes and hadron fluence by counting SEUs in SRAM memories. Description of the sensors can be found in [2]. In the LHC underground areas where high radiation levels are expected, the memories are biased at $5 \mathrm{~V}$. In order to increase the SEU cross section of the memories mostly in the shielded areas, the RadMon detector's memories are set to $3 \mathrm{~V}$, which is still within the specifications of the memory.

The high-energy hadron SEU cross-sections of the memories were measured in proton beams in PSI [3] and in UCL [4] as presented in this paper. The thermal neutron cross-section was measured in the epithermal neutron beam in NRI Rez

Manuscript received September 17, 2010; revised December 09, 2010 and January 05, 2011; accepted January 05, 2011. Date of publication March 10, 2011; date of current version June 15, 2011.

D. Kramer, M. Brugger, C. Pignard, K. Roeed, G. Spiezia, and T. Wijnands are with CERN, Geneva 23, CH-1211 Switzerland (e-mail: daniel.kramer@cern.ch).

V. Klupak and L. Viererbl are with NRI Rez, Prague, Czech Republic.

Color versions of one or more of the figures in this paper are available online at http://ieeexplore.ieee.org.

Digital Object Identifier 10.1109/TNS.2011.2105891 near Prague. The beam is mainly used by boron neutron capture therapy (BNCT) experiments. For our measurements, a polyamide moderator was used to increase the thermal neutron fluence rate.

The SEE susceptibility of some of the key LHC underground electronic systems was estimated using mostly $60 \mathrm{MeV}$ proton beams or mixed radiation field test areas around fixed targets, but no dedicated thermal neutron tests were conducted. Many systems and components were purchased in 1998 and may contain unknown amounts of ${ }^{10} \mathrm{~B}$. According to the recent simulation campaign [5], the thermal neutron part of the spectra in the LHC underground areas dominates the high energy hadron fluence by a factor of few to several hundreds. Hence the effect of the thermal neutrons can present a considerable additional risk to the operation of the LHC. The knowledge of the actual ratio of thermal neutrons to high energy hadrons therefore is an important ingredient for the characterization of the radiation field and the possible impact on equipment failures.

\section{B. Thermal Field Estimation Method}

When lowering the biasing voltage of an SRAM cell, the critical charge is expected to decrease linearly. The SEU cross-section is then expected to increase by the same fraction. However, when exposed to a thermal neutron beam, the ratio of measured RadMon sensitivities at different voltages was largely exceeding the simple reduction in critical charge. Based on this observation, it is expected that both fragments produced during the thermal neutron capture reaction within the memory can upset the SRAM cell and the importance of each contribution depends on the bias voltage. Hence changing the voltage will produce a nonlinear increase in low energy neutron cross-section as presented in the following. Based on this, it is proposed to combine the RadMon SRAM SEU measurements at two different voltages to estimate the ratio between the thermal neutron and high energy hadron fluence.

\section{EXPERIMENTAL SETUP}

\section{A. SRAM Memories}

The 4 Mbit SRAM used as a hadron fluence detector in the LHC RadMon is the Toshiba TC554001AF-70L with 0.4 um feature size. One detector contains $2 \mathrm{MB}$ of memory, which is read entirely at a rate of $1 \mathrm{~Hz}$, while the access time is maintained at $70 \mathrm{~ns}$. During the cycle, one Byte is read, compared to the reference (which is zero for all the standard RadMons) and then the reference is written to the corresponding Byte. For the measurements, the memories were biased between $3 \mathrm{~V}$ and $5 \mathrm{~V}$ 
TABLE I

Fluence Rates Measured at the TEST Location In the Wide SPECTRUM NEUTRON BEAM OF THE NuClEAR REACTOR IN NRI REZ NEAR PRAGUE

\begin{tabular}{lccc}
\hline \hline Neutron type & $\begin{array}{c}\text { Fluence rate } \\
{\left[\mathrm{cm}^{-2} / \mathrm{s}\right]}\end{array}$ & $\begin{array}{c}\text { Uncertainty } \\
{\left[\mathrm{cm}^{-2} / \mathrm{s}\right]}\end{array}$ & Energy range \\
\hline Thermal & $1.0610^{7}$ & $4.010^{5}$ & $<0.5 \mathrm{eV}$ \\
Epithermal & $1.6710^{7}$ & $1.910^{6}$ & $0.5 \mathrm{eV}$ to $100 \mathrm{keV}$ \\
Fast & $1.4410^{6}$ & $1.110^{6}$ & $>100 \mathrm{keV}$ \\
\hline
\end{tabular}

via a manually adjusted power supply in the experimental control room. The voltage was changed in steps during a continuous run and the transient periods were excluded from the analysis. In case of standard RadMon detectors as installed in the LHC, the memories can be biased either to $5 \mathrm{~V}$ or to $3 \mathrm{~V}$ by selecting the appropriate voltage regulator via manual switches.

\section{B. Neutron Test Beam}

The thermal neutron cross section of the RadMon memories was measured in the wide spectrum neutron beam in the BNCT beam line of the Nuclear Research Institute (NRI) in Rez near Prague. Neutrons are produced by the 10 MW LVR-15 [6] reactor with a filtering and collimating beam line equipped with a shutter. This allows access to the test area without stopping the reactor.

The relative beam intensity was measured with two fission chambers inside the collimator. The absolute fluence was measured with activation foils at the place of the detector immediately after the end of the tests.

The results of the activation foil analysis are presented in Table I for three different energy ranges. The thermal fluence rate is comparable to the epithermal one, whereas the fast neutrons above $100 \mathrm{keV}$ present less than $10 \%$ of the spectrum. The BNCT neutron beam is accompanied by gamma radiation with a dose rate of about $0.5 \mathrm{~Gy} / \mathrm{h}$ at the test location. The total measurement time was only several hours so the integrated dose was well below the design tolerance of the detector of 200 Gy reported in [7].

\section{MeAsurement Results}

\section{A. Raw Data}

The RadMon detector was placed in the neutron beam as shown in Fig. 1 and the number of SEUs was read out every second. During the continuous measurement, the voltage was changed in steps of $0.25 \mathrm{~V}$ remotely from $5 \mathrm{~V}$ down to $3 \mathrm{~V}$ (see Fig. 2). In a second step, the detector was wrapped in a $1 \mathrm{~mm}$ thick Cd sheet and placed back into the beam. The voltage scan was then repeated. The shape of the response is very similar, only the absolute level is lower, as it can be seen in Fig. 3. The $\mathrm{Cd}$ sheet very efficiently removes the thermal neutrons from the spectra, thus allowing to discriminate between a pure low-energy neutron component $(<0.5 \mathrm{eV})$ and the total neutron fluence.

In this respect, for each constant voltage setting, the difference in the fitted slopes of the raw data without and with $\mathrm{Cd}$ divided by the thermal neutron fluence rate gives the thermal neutron SEU cross section for the corresponding voltages.

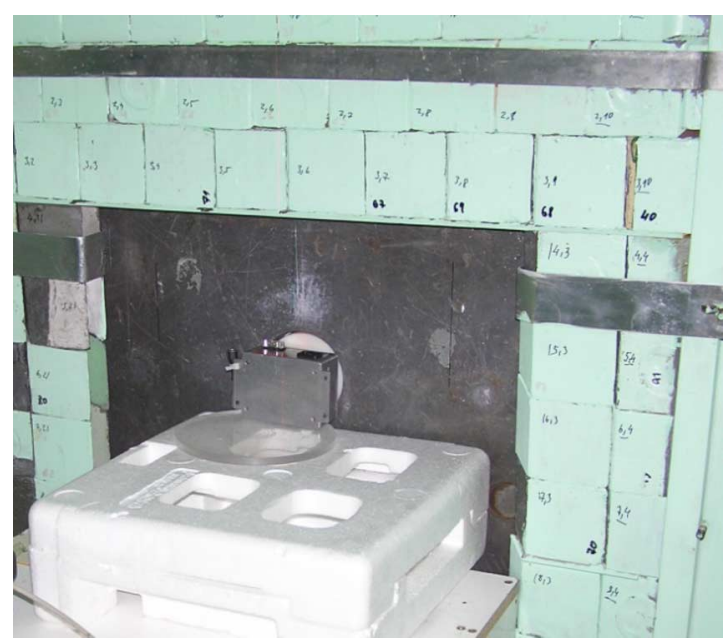

Fig. 1. Positioning of the RadMon detector in the BNCT beam line.

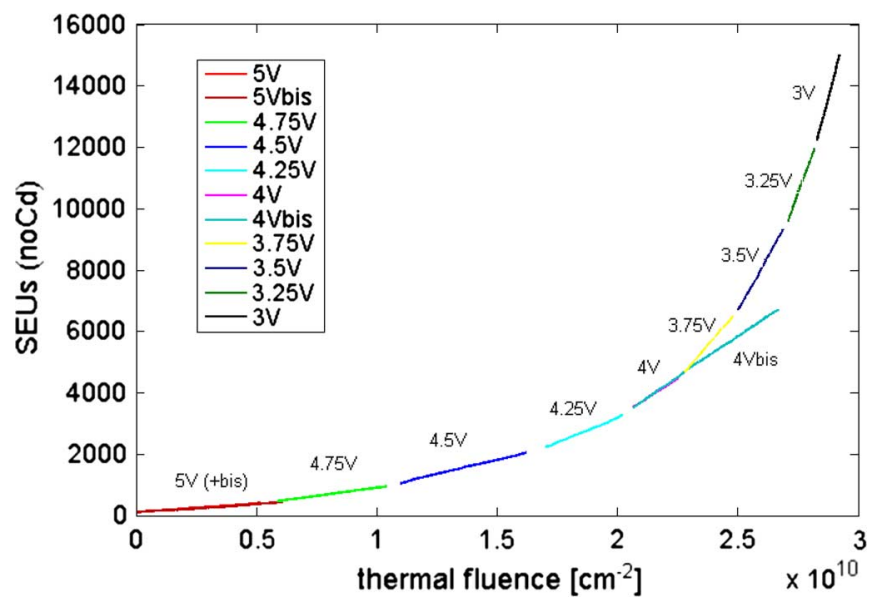

Fig. 2. Measured cumulative SEU counts at different voltages without $\mathrm{Cd}$ wrapping. Repeated measurements are marked bis.

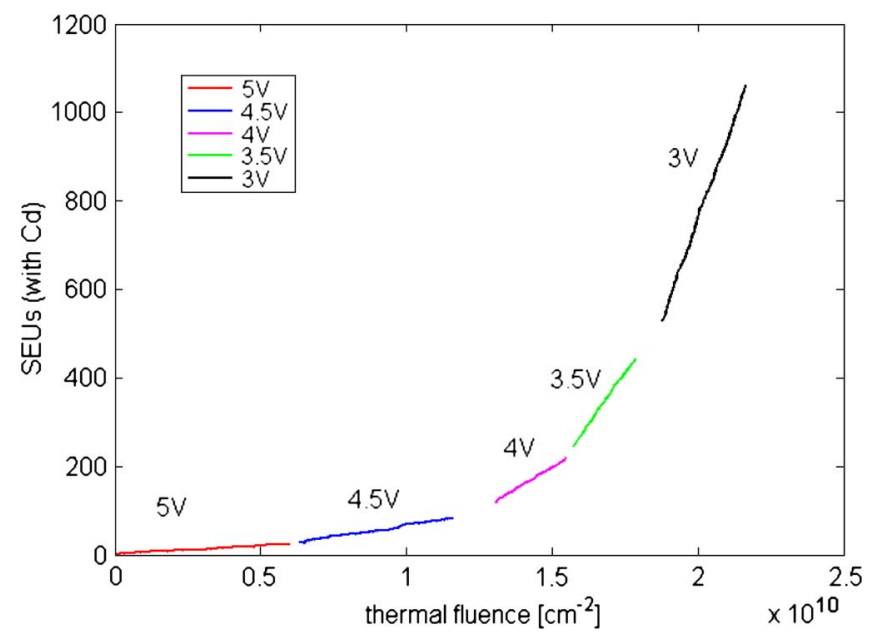

Fig. 3. Measured cumulative SEU counts at different voltages inside the $\mathrm{Cd}$ wrapping. The thermal fluence refers to the measured field without $\mathrm{Cd}$ absorber.

\section{B. Proton Cross-Sections}

The high energy proton cross sections of the RadMon SRAM were measured at UCL cyclotron in 2006 and recently at PSI at 


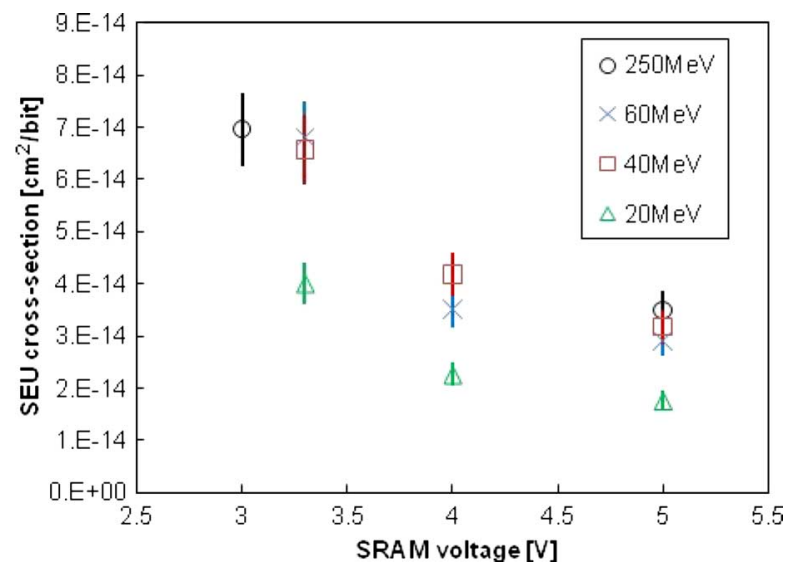

Fig. 4. Measured SEU cross sections of the Toshiba TC554001AF-70L SRAM obtained in 2010 at PSI $(250 \mathrm{MeV})$ and in 2006 at UCL for different biasing voltages.

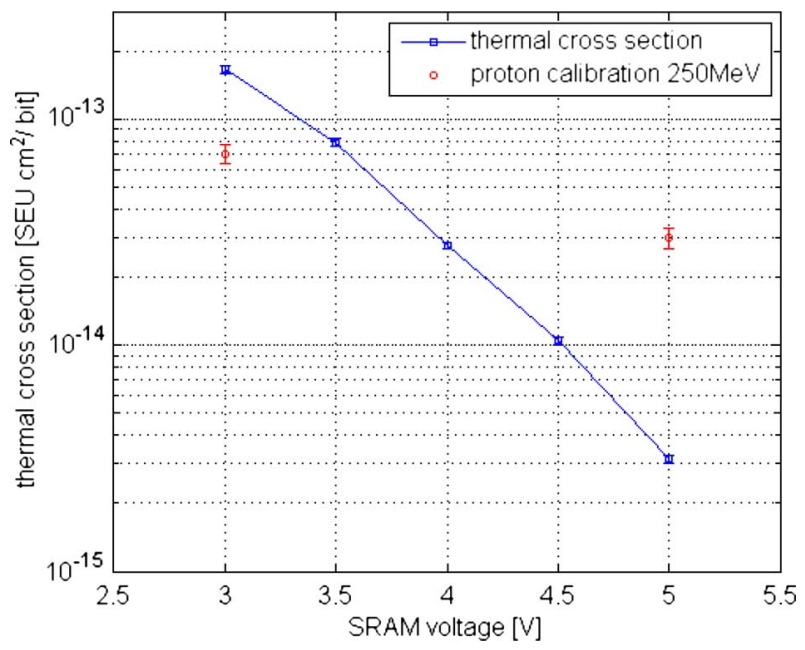

Fig. 5. Measured thermal neutron cross sections per bit for different biasing voltages. The round points indicate the results obtained with $60 \mathrm{MeV}$ proton beams.

$250 \mathrm{MeV}$. These tests were conducted for both cases on the production batch number 0443MAD using the standard RadMon detectors and with an equivalent test setup as described above.

The voltage dependence of the cross-sections at different energies is presented in Fig. 4.

\section{Resulting Cross-Sections}

The thermal neutron SEU cross section increases nearly exponentially when the voltage is lowered as it can be seen in Fig. 5. Table I shows that the thermal cross section measured at $5 \mathrm{~V}$ is about 10 times lower than the SEU cross section obtained with high energy hadron beams. On the other hand, the 3 $\mathrm{V}$ thermal cross section is 2.4 times greater than the high energy one.

It shall be noted that the result obtained for the $5 \mathrm{~V}$ biasing is in good agreement with the previously measured value for this memory type (i.e., $2.7 \cdot 10^{-15} \mathrm{~cm}^{2}$ ) at NPL [8].

The ratio between the $3 \mathrm{~V}$ and $5 \mathrm{~V}$ thermal SEU cross section is about 50 which is significantly higher than the ratio of $\sim 2.4$ obtained with $60 \mathrm{MeV}$ proton beams.

\section{Discussion of the Results}

The thermal neutron induced SEU is most likely produced by the ${ }^{10} \mathrm{~B}(\mathrm{n}, \alpha)^{7} \mathrm{Li}$ reaction. The exponential dependence on the voltage suggests that at $5 \mathrm{~V}$ the alpha particle which carries more energy (it can deposit at most $\sim 65 \mathrm{fC}$ in silicon) and has a higher penetration depth (in the order of $5 \mu \mathrm{m}$ ) is the dominating recoil of the reaction. When the voltage is lowered, the critical charge decreases accordingly, thus the Lithium ion starts also contributing to the SEU process even though its range in silicon is significantly shorter (about $2.5 \mu \mathrm{m}$ ) and it can produce only about $37 \mathrm{fC}$ of total charge.

The shape of the SEU counts versus the voltage curve is identical whether the detector is wrapped with $\mathrm{Cd}$ or not, only the absolute level is about 15 times smaller. This shows that the process responsible for the SEUs above the Cd cutoff is the same as for the thermal neutrons. The contribution of the fast neutrons from the BNCT beam is therefore considered to be negligible. A possible contribution could still come from the thermal neutrons penetrating along the incoming detector cables, where the $\mathrm{Cd}$ shielding was the weakest. This contribution is however expected to be $<1 \%$ (based on phase space assumptions and first principles).

\section{E. Thermal Neutron Field Estimation With RadMon Detectors}

It is therefore proposed, that the difference of the observed sensitivities as function of device voltage can be exploited in mixed field environments to determine the actual thermal neutron content of the spectra, if the measurement is performed at both voltages. If only one voltage is employed, the measured cross sections have to be analyzed together with the assumed ratio between the relevant fields ( $r$-factor as obtained through simulations or additional measurements) in order to be able to separate the thermal neutron fluence contribution.

In the following, the thermal neutron to high energy hadron fluence ratio $r$ is taken as

$$
r=\frac{\Phi_{t h}}{\Phi_{H E}} .
$$

We assume that part of the SEUs is caused either by charged hadrons with energy above the coulomb barrier ( $\sim 20 \mathrm{MeV}$ for protons) or neutrons above few $\mathrm{MeV}$. In order to deposit enough energy to upset a memory cell, heavy ions have to reach the corresponding sensitive volume. At LHC the heavy ions are produced by hadronic interactions, mainly induced by protons, pions and neutrons [9], while direct fragments coming from the beam line during ion runs are not expected to reach the RadMon detectors.

Another part of the SEUs is induced by thermal neutron capture process as discussed above.

The contribution of the thermal neutrons to the RadMon signal in a mixed radiation field environment can be determined if the energy spectrum is known. Currently at the LHC, the ratio $r$ is usually obtained through dedicated FLUKA Monte-Carlo simulations [10], [11]. Assuming a known ratio $r$, the fluence 
TABLE II

MEASUREd SEU CROSS SECTIONS

\begin{tabular}{lcccc}
\hline \hline $\begin{array}{l}\text { Field } \\
\text { type }\end{array}$ & $\begin{array}{c}\text { cross-section } \\
\text { at } 5 \mathrm{~V} \text { bias } \\
{\left[\mathrm{cm}^{2} / \mathrm{bit}\right]}\end{array}$ & $\begin{array}{c}\text { uncertainty } \\
{\left[\mathrm{cm}^{2} / \mathrm{bit}\right]}\end{array}$ & $\begin{array}{c}\text { cross-section } \\
\text { at } 3 \mathrm{~V} \text { bias } \\
{\left[\mathrm{cm}^{2} / \mathrm{bit}\right]}\end{array}$ & $\begin{array}{c}\text { uncertainty } \\
{\left[\mathrm{cm}^{2} / \mathrm{bit}\right]}\end{array}$ \\
\hline $\begin{array}{l}\text { Thermal } \\
\text { High } \\
\text { energy } \\
\text { hadrons }\end{array}$ & $3.110^{-15}$ & $310^{-16}$ & $1.710^{-13}$ & $210^{-14}$ \\
\hline \hline
\end{tabular}

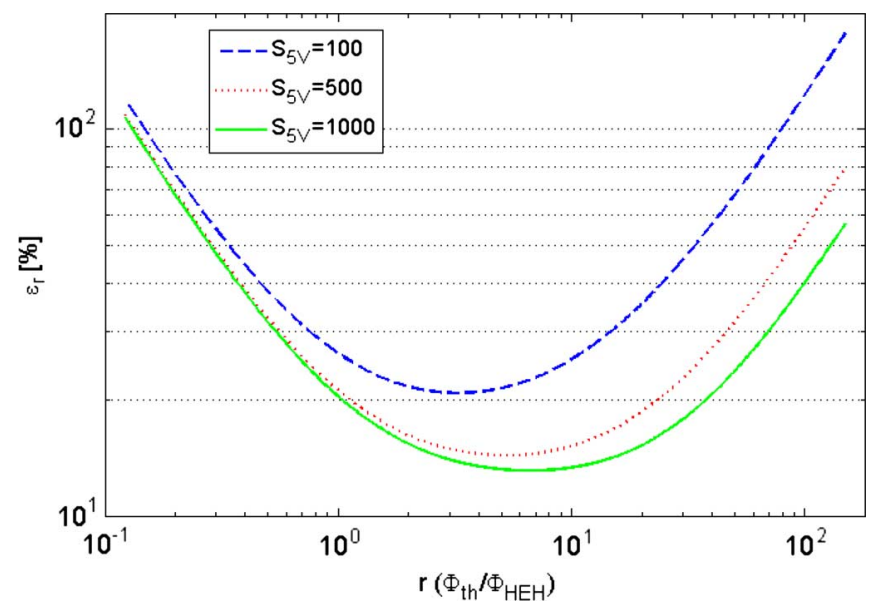

Fig. 6. Relative error of $r$ (ratio of thermal neutron to high energy hadron fluence) plotted vs. the value of $r$. The absolute number of counts obtained under $5 \mathrm{~V}$ bias is used as a parameter for the curves.

of high energy hadrons can be estimated based on the number of SEUs measured at $3 \mathrm{~V} S_{3} \mathrm{~V}$ as follows:

$$
\Phi_{H E}^{3 V}=\frac{S_{3 V}}{\sigma_{t h}^{3 V} r+\sigma_{H E}^{3 V}} .
$$

The thermal neutron fluence at the same voltage is calculated similarly

$$
\Phi_{t h}^{3 V}=\frac{S_{3 V}}{\sigma_{t h}^{3 V}+\frac{\sigma_{H E}^{3 V}}{r}} .
$$

Based on the above formulas and, in case of unknown spectra, the ratio $r$ can be calculated using the known cross-sections from Table II and two measurements of SEU counts at the corresponding voltages

$$
r=\frac{\sigma_{H E}^{3 V} S_{5 V}-\sigma_{H E}^{5 V} S_{3 V}}{\sigma_{t h}^{5 V} S_{3 V}-\sigma_{t h}^{3 V} S_{5 V}} .
$$

Using this approach it should be noted that in case of a pure thermal neutron field, $r$ tends to infinity, whereas the ratio of the counts measured under the same conditions converges to $\sim 50$. On the other hand, if the field was fully dominated by the high energy part, $r$ would then converge towards 0 and the ratio of SEU counts to $\sim 2.4$. If there were no measurement errors and no SEU sensitivity in other parts of the spectra, the ratio of the $3 \mathrm{~V} / 5 \mathrm{~V}$ counts in the same conditions should range between $\sim 2.4$ and $\sim 50$.

\section{F. Sensitivity Analysis}

The error of the ratio $r$ depends on all six parameters of (4). Only the statistical error is assumed for the number of counts as listed in Table II. The cross-sections are assumed to be known within $10 \%$.

When reaching very low values of $r$, the error is dominated by the uncertainty of the $5 \mathrm{~V}$ cross-sections. On the other hand, when reaching high values of $r$, the error is diverging due to high derivation of $r$.

The error propagation for $r$ was calculated using the partial differentials as follows:

$$
\begin{aligned}
\Delta r^{2}= & \left(\frac{\partial r}{\partial S^{5 V}} \Delta S_{5 V}\right)^{2}+\left(\frac{\partial r}{\partial S^{3 V}} \Delta S_{3 V}\right)^{2} \\
& +\left(\frac{\partial r}{\partial \sigma_{H E}^{3 V}} \Delta \sigma_{H E}^{3 V}\right)^{2}+\left(\frac{\partial r}{\partial \sigma_{H E}^{5 V}} \Delta \sigma_{H E}^{5 V}\right)^{2} \\
& +\left(\frac{\partial r}{\partial \sigma_{t h}^{3 V}} \Delta \sigma_{t h}^{3 V}\right)^{2}+\left(\frac{\partial r}{\partial \sigma_{t h}^{5 V}} \Delta \sigma_{t h}^{5 V}\right)^{2} .
\end{aligned}
$$

The error is a function of $\mathrm{S}_{3} \mathrm{~V}$ and $\mathrm{S}_{5} \mathrm{~V}$ given the cross-sections are known as well as their uncertainties.

The resulting error of $r$ versus the absolute value of $r$ is presented in Fig. 6. It is clear that in order to obtain an $r$ with an uncertainty of less than $20 \%$, one has to reach a few hundred counts at $5 \mathrm{~V}$ bias and $r$ has to range between 1 and few tens (i.e. up to 25 for 500 counts at $5 \mathrm{~V}$ ). With higher numbers of counts, the overall error is dominated by the uncertainty of the cross-sections.

\section{COMPARISON With FluKa Simulations}

\section{A. Test Measurements in CNRAD}

During dedicated irradiation tests of LHC electronics the RadMon detectors were placed in the mixed field LHC electronics test area CNRAD [12] which is located close to the CNGS [13], [14] target chamber. The CNGS experiment uses a high current $450 \mathrm{GeV}$ proton beam hitting a graphite target. Thanks to the very stable beam conditions, the integrated radiation levels scale with the number of protons on target (pot), thus allowing to create mixed particle energy spectra similar to the critical areas for electronics in the LHC. At CNRAD, several locations were measured at $3 \mathrm{~V}$ and $5 \mathrm{~V}$ to characterize the ongoing radiation tests.

In addition, the particle energy spectra were simulated at the test locations with the FLUKA code. The CNRAD simulations are very complex due to the size of the geometry and the number of details to be taken into account. A few shortcomings are known (i.e. the water content of the concrete is not known and the round wall edges are not fully reproduced in the model), therefore the error on the thermal fluence data is assumed to be at least a factor of a few (i.e., 2 to 3 ).

To measure the latter component also by other means than the RadMons, several locations were equipped with $0.1 \mathrm{~mm}$ thick activation gold foils in order to probe the thermal neutron fluence using the known cross-section of the capture reaction (n, $\left.{ }^{197} \mathrm{Au}\right){ }^{198} \mathrm{Au}$. 
TABLE III

FluENCE RATIO ObTaINED From MEASURED SEUS (COLUMN 7) IN CNGS AND CERF (C1 to C6) COMPaRED tO THE Simulated Fluence Ratios

\begin{tabular}{lccccccc}
\hline \hline $\begin{array}{l}\text { Area } \\
\text { code }\end{array}$ & $\begin{array}{c}\text { Sim. } \\
r^{\mathrm{A}}\end{array}$ & $\Delta r^{A}$ & $\begin{array}{c}\text { Sim. } \\
r^{\mathrm{B}}\end{array}$ & $\Delta r^{B}$ & $\begin{array}{c}r^{\mathrm{B}} \text { corrected by } \\
\text { Au activation } \\
\text { measurement }\end{array}$ & $\begin{array}{c}\text { Measured } \\
r\end{array}$ & $\Delta r$ \\
\hline 455 & 41 & 0.8 & 23 & 0.5 & 11 & 7.2 & 2.1 \\
456 & 90 & 1.6 & 42 & 0.7 & 9 & 6.0 & 1.7 \\
464 & 10 & 1.2 & 8.3 & 0.8 & & 1.4 & 0.39 \\
467 & 57 & 1.2 & 33 & 0.6 & 10 & 12 & 4.4 \\
S1 & 650 & 29 & 162 & 4.4 & 31 & 25 & 15 \\
S2 & 560 & 41 & 152 & 7.2 & 29 & 31 & 20 \\
S4 & 730 & 40 & 197 & 6.5 & 95 & 34 & 24 \\
\hline C1 & 5.7 & 0.05 & 2.6 & 0.02 & & 2.5 & 0.8 \\
C2 & 2.4 & 0.02 & 1.2 & 0.01 & & 0.92 & 0.27 \\
C3 & 0.67 & 0.004 & 0.36 & 0.002 & & 0.33 & 0.06 \\
C4 & 0.64 & 0.004 & 0.46 & 0.003 & & 0.53 & 0.18 \\
C5 & 0.46 & 0.004 & 0.42 & 0.003 & & 0.35 & 0.06 \\
C6 & 0.26 & 0.002 & 0.25 & 0.002 & & 0.22 & 0.05 \\
\hline \hline
\end{tabular}

${ }^{\mathrm{A}} \mathrm{High}$ energy hadron threshold is set to $20 \mathrm{MeV}$ in FLUKA

${ }^{\mathrm{B}} \mathrm{Same}$ as ${ }^{\mathrm{A}}$ but the neutron threshold is set to $5 \mathrm{MeV}$ in FLUKA

Columns marked with $\Delta$ represent the simulation or measurement errors

\section{B. Test Measurements at CERF}

In order to obtain measurements with lower thermal neutron component, a set of measurements was performed at the CERF facility [15] using a mixed radiation field around a $\mathrm{Cu}$ target hit by a $120 \mathrm{GeV} / \mathrm{c}$ proton/pion beam. The radiation fields of the facility are very well characterized and can be therefore used as a very good reference. The simulated spectra were successfully benchmarked with different instruments and activation foils [16]-[19]. Our CERF measurements however suffer from higher statistical uncertainties due to limited beam intensities. The number of counts obtained at each position ranges between 70 and 1000 for different bias voltages. The corresponding CERF FLUKA simulations have small statistical uncertainties (below 1\%) thanks to the relatively simple geometry.

The CERF internal areas are marked C1 to C6 in Table III.

\section{Results}

The ratio $r$ was obtained using (4) from the measured counts and is shown in the seventh column of Table III.

The scoring threshold for high energy hadrons in the FLUKA simulations was set to $20 \mathrm{MeV}$, which is not a physical barrier for neutrons as they don't have to overcome the coulomb barrier. Recently conducted monoenergetic neutron beam tests performed in CEA Valduc and in PTB [20] suggest that the cross-section starts to decrease at about $10 \mathrm{MeV}$ and significantly contributes down to $5 \mathrm{MeV}$. Therefore, for comparison, additional simulations used the neutron scoring threshold lowered to $5 \mathrm{MeV}$ (fourth column in Table III). From the simulation point of view, the neutron SEU cross-section is then assumed constant from $5 \mathrm{MeV}$ to $20 \mathrm{MeV}$ and equal to the hadron SEU cross-section above $20 \mathrm{MeV}$.

The simulations with lower threshold (marked as ${ }^{B}$ ) yield results considerably closer to the ratios measured with the SRAM data. The best agreement with the simulations is obtained in the
CERF areas, where thermal ratios are moderate and the environment is very well known. On the other hand, the CNRAD simulations yield significantly higher thermal ratios than the measurements. Verification measurements conducted with $\mathrm{Au}$ activation foils have revealed a significant overestimation of the simulated thermal fluence in the CNRAD test areas. For example in case of $S 1$ a kicker magnet installation lowers the thermal flux and wasn't included in the model. The column 6 of Table III presents the thermal fluence measured with activation foils divided by high energy hadron fluence simulated with the same thresholds as in $r^{B}$.

The worst disagreement between the simulations and measurements is observed for areas 456 and 464, which are in high gradient locations (behind wall edges), thus particularly sensitive to both the concrete composition as well as the geometry of the edges. Nevertheless, when $r^{B}$ of the area 456 is corrected by the activation foil, it gets considerably closer to the measured value. The area $S 4$ shows a high thermal neutron content, but is not correctly reproduced by the measurements even if the simulated value is corrected by the activation foil result. The disagreement is possibly caused by geometry inaccuracies.

\section{CONCLUSION}

The thermal neutron SEU cross-section of the RadMon SRAM memories was measured at different voltages in an epithermal beam and compared to the previously obtained high energy proton and neutron beam results. The thermal cross section at $3 \mathrm{~V}$ was found to be 50 times higher than at $5 \mathrm{~V}$ under the same experimental conditions.

Based on the observed large difference between the thermal neutron/high energy hadron cross section ratios at $3 \mathrm{~V}$ and 5 $\mathrm{V}$, a method to estimate the thermal neutron and the high energy hadron fluence separately from a single memory type is proposed. In order to achieve greater precision of the method, the neutron SEU cross sections from several $\mathrm{MeV}$ to $20 \mathrm{MeV}$ will have to be measured. The error of the method is diverging fast in fields with large thermal neutron or high energy hadron dominance but can give satisfactory results at moderate thermal neutron to high energy hadron ratios up to few tens.

\section{OUTLOOK}

The cross section measurements of the Toshiba SRAM were recently performed with monoenergetic neutron beams produced by DD or DT reactions at PTB and the analysis is ongoing. The data should clarify the neutron sensitivity between 5 and $20 \mathrm{MeV}$ in order to better set the scoring range of the simulations to match the detector response. Two TIMEPIX [21] detectors with multiple converters were placed in the CNRAD test facility in order to compare their mixed field response to the RadMons and the simulations.

\section{ACKNOWLEDGMENT}

The authors would like to thank the CNGS collaboration for providing the FLUKA simulation model of the experimental area and for hosting the CNRAD facility, the CERN Radio Protection group for support of the tests, and the CERN FLUKA team. 


\section{REFERENCES}

[1] P. Collier et al., LHC Design Rep. Geneva, Switzerland, CERN-2004003, 2004.

[2] T. Wijnands, C. Pignard, and R. Tesarek, "An on line radiation monitoring system for the LHC machine and experimental caverns," in Proc. 12th Workshop Electron. LHC Future Exp., Valencia, Spain, 2006.

[3] Proton Irradiation Facility Paul Scherrer Inst.. Villigen, Switzerland [Online]. Available: http://pif.web.psi.ch/

[4] G. Berger, G. Ryckewaert, R. Harboe-Sorensen, and L. Adams, "CYCLONE-A multipurpose heavy ion, proton and neutron SEE test site," in Proc. RADECS, 1997.

[5] K. Roeed et al., "FLUKA simulations for SEE studies of critical LHC underground areas," in Proc. RADECS, 2010.

[6] J. Burian et al., "LVR-15reactor epithermal neutron beam parameters-Results and measurements," Appl. Radiat. Isot., vol. 67, pp. S202-S205, 2009.

[7] T. Wijnands and C. Pignard, "Radiation tolerant commercial-off-the-shelf components for the remote readout of RADFETs and PIN diodes," in Proc. RADECS, 2005.

[8] C. S. Dyer, S. N. Lucas, C. Anderson, A. D. Frydland, and R. T. Green, "An experimental study of single event effects induced in commercial SRAMs by neutrons and protons from thermal energies to $500 \mathrm{MeV}$," in Proc. RADECS, 2003.

[9] M. Huhtinen and F. Faccio, "Computational method to estimate single event upset rates in an accelerator environment," Nucl. Instrum. Methods Phys. Res. A, Accel. Spectrom. Detect. Assoc. Equip., vol. 450, no. 1, pp. 155-172, 2000.

[10] G. Battistoni, S. Muraro, P. R. Sala, F. Cerutti, A. Ferrari, S. Roesler, A. Fassò, and J. Ranft, "The FLUKA code: Description and benchmarking," in Proc. Hadronic Shower Simulation Workshop, 2007, pp. $31-49$.

[11] A. Fassò, A. Ferrari, J. Ranft, and P. R. Sala, FLUKA: A Multi-Particle Transport Code CERN-2005-10, 2005, INFN/TC_05/11, SLAC-R-773.
[12] CNRAD [Online]. Available: http://cngs-rad-facility.web.cern.ch/ cngs-rad-facility

[13] CERN Neutrinos to Gran Sasso [Online]. Available: http://proj-cngs. web.cern.ch/proj-cngs/

[14] E. Gschwendtner et al., "Performance and operational experience of the CNGS facility," in Proc. 1st Int. Particle Accelerator Conf., Kyoto, Japan, May 23-28, 2010.

[15] A. Mitaroff and M. Silari, "The CERN-EU high-energy Reference Field (CERF) facility for dosimetry at commercial flight altitudes and in space," Radiat. Protection Dosimetry, vol. 102, pp. 7-22, 2002.

[16] C. Theis, D. Forkel-Wirth, D. Perrin, S. Roesler, and H. Vincke, "Simulation and measurements of the response of an air ionisation chamber exposed to a mixed high-energy radiation field," Radiat. Protection Dosimetry, vol. 116, no. 1-4, pp. 380-386, 2005.

[17] S. Mayer, D. Forkel-Wirth, M. Fuerstner, H. G. Menzel, M. J. Mueller, D. Perrin, C. Theis, and H. Vincke, "Response of neutron detectors to high-energy mixed radiation fields," Radiat. Protection Dosimetry, vol. 125, no. 1-4, pp. 289-292, 2007.

[18] L. Sarchiapone, M. Brugger, B. Dehning, D. Kramer, M. Stockner, and V. Vlachoudis, "FLUKA Monte Carlo simulations and benchmark measurements for the LHC beam loss monitors," Nucl. Instrum. Methods Phys. Res. A, Accel. Spectrom. Detect. Assoc. Equip., vol. 581, no. 1-2, pp. 511-516, 2007.

[19] M. Brugger, H. Khater, S. Mayer, A. Prinz, S. Roesler, L. Ulrici, and H. Vincke, "Benchmark studies of induced radioactivity produced in LHC materials, PART I: Specific activities," Radiat. Protection Dosimetry, vol. 116, no. 1-4, pp. 6-11, 2005.

[20] S. Rottger et al., "The PTB neutron reference fields (PIAF)—Quasimonoenergetic neutron reference fields in the energy range from thermal to $200 \mathrm{MeV}$," in Proc. AIP Conf., 2009, p. 375.

[21] X. Llopart, R. Ballabriga, M. Campbell, L. Tlustos, and W. Wong, "Timepix, a $65 \mathrm{k}$ programmable pixel readout chip for arrival time, energy and/or photon counting measurements," Nucl. Instrum. Methods Phys. Res. A, Accel. Spectrom. Detect. Assoc. Equip., vol. 581, no. 1, pp. 485-494, 2007. 